\title{
Tumor filodes de la mama. Caracterización clínica e histopatológica de 39 casos $^{*}$ Breast phyllodes tumors. Review of medical records of 39 patients
}

\author{
Drs. JUAN A. PÉREZ P.1,3, GONZALO SÁNCHEZ C.1,3, JOHN BOHLE O. ${ }^{1,3}$, MARÍA TERESA \\ POBLETE S. ${ }^{2,4}$, MARCELA HERNÁNDEZ H.1,3, DANIELA MASSRI E. ${ }^{5}$ \\ ${ }^{1}$ Servicio de Cirugía, Hospital Regional Valdivia. ${ }^{2}$ Servicio de Anatomía Patológica, Hospital Regional Valdivia. \\ 3 Instituto de Cirugía, Facultad de Medicina, Universidad Austral de Chile. ${ }^{4}$ Instituto de Histopatología, Facultad de \\ Medicina, Universidad Austral de Chile. ${ }^{5}$ Interna de Cirugía. Facultad de Medicina, \\ Universidad Austral de Chile. Valdivia Chile.
}

\begin{abstract}
RESUMEN
Introducción: Los tumores filodes mamarios son neoplasias poco frecuentes habitualmente benignas. El diagnóstico diferencial principal es el fibroadenoma. El objetivo de este trabajo es caracterizar clínica e histopatológicamente a 39 mujeres tratadas en nuestro hospital. Material y Método: Estudio retrospectivo y descriptivo de fichas clínicas e informes histopatológicos de mujeres con tumores filodes de mama en el Hospital de Valdivia entre los años 1985 y 2006. Resultados: La edad promedio de las pacientes fue 44.4 años (extremos: 20 y 66 años). El tamaño promedio del tumor fue $8.3 \mathrm{~cm}$ (extremos: 2 y $28 \mathrm{~cm}$ ). En $27 \mathrm{se}$ realizó mamografía y en 13 ecotomografía, exámenes que orientaron al diagnóstico. Se obtuvo citología en 20 casos, siendo en 5 compatible con tumor fibroepitelial. El diagnóstico preoperatorio fue correcto en 23 pacientes. El tratamiento fue quirúrgico practicándose 29 mastectomías parciales, 7 mastectomías simples, 1 mastectomía simple extendida, 1 mastectomía de aseo y 1 mastectomía subcutánea. La histopatología concluyó 32 tumores benignos, 3 potencialmente malignos y 4 malignos. Un tumor maligno con margen infiltrativo y sobrecrecimiento estromal desarrolló metástasis pulmonares. El seguimiento promedio es 70 meses, registrándose una recidiva local y una metástasis pulmonar. Conclusiones: El diagnóstico de tumor filodes se sospecha en masas mamarias voluminosas circunscritas sin compromiso de linfonodos regionales. El tratamiento quirúrgico es efectivo siempre que se reseque la totalidad del tumor y un margen de tejido sano. El margen infiltrativo, sobrecrecimiento estromal y número de mitosis, pareciera asociarse con el desarrollo de recidiva tumoral y de metástasis sistémicas.
\end{abstract}

PALABRAS CLAVE: Neoplasias mamarias, tumor filodes mamario.

\section{SUMMARY}

Background: Breast phyllodes tumors are uncommon lesions that are usually benign. They must be differentiated from fibroadenoma. Aim: To report the clinical and pathological features of 39 women with breast phyllodes tumors. Material and Methods: Review of medical records of 39 women with breast phyllodes tumors, aged 20 to 66 years, treated in a regional hospital between 1985 and 2006. Results: A

*Recibido el 18 de diciembre 2006 y aceptado el 30 de enero de 2007.

Correspondencia: Dr. Juan Antonio Pérez P.

Arturo Günther 1664. Valdivia, Chile.

email: jperez@uach.cl 
mammography was performed in 27 women and an ultrasound in 13 . The diameter of the lesions ranged from 2 to $28 \mathrm{~cm}$. A cytological sample was obtained in 20 cases and in five it was compatible with a fibroepithelial tumor. The preoperative diagnosis was correct in 23 patients. Twenty nine women were subjected to a partial mastectomy, seven to a simple mastectomy, one to simple extended mastectomy, one to a cleaning mastectomy and one to a subcutaneous mastectomy. According to the pathological diagnosis, 32 tumors were benign, three were potentially malignant and four were malignant. One malignant tumor with infiltrated operative margins and estromal overgrowth, developed lung metastases. After a 70 month follow up, one patient, apart from the patient with metastases, had a local relapse. Conclusions: Phyllodes tumors should be suspected in voluminous breasts without local lymph node involvement. Surgical treatment is effective, when the whole tumor is excised.

\section{KEY WORDS: Phyllodes tumors, breast tumors, mastectomy.}

\section{INTRODUCCIÓN}

Los tumores filodes (TF) de la mama son neoplasias de estructura foliada, compuestas por tejido conectivo y elementos análogos al fibroadenoma del cual se diferencian por la mayor celularidad del tejido conectivo ${ }^{1}$. Representan el 0,3 a $1 \%$ de los tumores mamarios y el 1 a $2,5 \%$ de los tumores fibroepiteliales ${ }^{2}$. La incidencia anual en la población general es de 2,1 x millón de habitantes, aumentando en la población latina a 2,8 x millón de personas ${ }^{3}$. Aparecen usualmente entre los 35 y 55 años, y es rara su presentación en varones ${ }^{4}$.

Los TF se dividen en benignos, potencialmente malignos (borderline) y malignos ${ }^{1}$.

Clínicamente se manifiestan como tumores voluminosos, habitualmente mayores de $5 \mathrm{~cm}$, indoloros, consistencia firme, superficie abollonada o lobulada, bien delimitados, móviles y sin compromiso de piel ni planos profundos ${ }^{5}$. Se asocian con linfonodos axilares inflamatorios en un 17\% y metastáticos en un 1\%². La diseminación sistémica es rara y afecta principalmente a pulmones, huesos, hígado y cerebro6.

El diagnóstico diferencial debe realizarse principalmente con el fibroadenoma mamario ${ }^{2}$.

El estudio del TF puede precisar de mamografía, ecotomografía mamaria y de citología 5 .

El tratamiento es quirúrgico $0^{4,5,7-9}$ y la extensión de la cirugía depende de las características del TF y de la mama afectada. No se recomienda la disección nodal electiva dado el bajo compromiso metastático ${ }^{10}$. En TF malignos se ha utilizado quimioterapia y/o radioterapia complementaria sin resultados alentadores ${ }^{6,11}$.

El seguimiento se realiza mediante evaluaciones clínicas periódicas. En TF malignos se sugiere practicar radiografía de tórax, ecotomografía abdominal, cintigrama óseo y eventualmente tomografía cerebral 6 .

El objetivo de este trabajo es presentar una caracterización clínica y patológica de pacientes portadoras de tumor filodes mamario manejadas en el Hospital Regional de Valdivia en un periodo de 20 años

\section{MATERIAL Y METODO}

Revisión retrospectiva de registros clínicos e histopatológicos de pacientes con diagnóstico de tumor filodes de mama durante el período comprendido entre enero 1985 y enero de 2006.

Las variables analizadas fueron edad, sexo, antecedentes mórbidos, método de detección del tumor, tipo de crecimiento, tiempo entre la detección y la consulta, compromiso de linfonodos axilares, exámenes practicados previo a la cirugía (mamografía, ecotomografía mamaria, citología), diagnóstico preoperatorio, tipo de cirugía realizada, tratamiento complementario y evolución de las pacientes.

Se analizaron las placas histológicas de las 39 pacientes. En el estudio anatomopatológico se identificaron los siguientes caracteres macro y microscópicos: tamaño tumoral, margen tumoral, grado de celularidad estromal, pleomorfismo estromal nuclear, actividad mitótica, sobrecrecimiento estromal, presencia o ausencia de necrosis, quistes y estroma especializado. Estos parámetros se estudiaron acorde a los criterios establecidos por Hawkins ${ }^{6}$.

a) Tamaño tumoral: Diámetro transverso máximo al examen microscópico en $\mathrm{cm}$.

b) Margen tumoral: expansivo, infiltrativo, no evaluable.

c) Grado de celularidad estromal: (+) escaso, $(++)$ intermedio, $(+++)$ densamente celular.

d) Pleomorfismo estromal: sobre la base del diámetro nuclear máximo, un incremento de 30 más veces esta medida fue considerado como severo.

e) Mitosis: Medición en el centro del tumor, el número de mitosis por 10 campos de alto aumento. 
f) Sobrecrecimiento estromal: Presencia de áreas con solo células estromales, sin elementos glandulares que llenen un campo de bajo aumento (magnificación 4x): 1 campo (+), 2 campos vecinos $(++), 3$ campos vecinos (+++). tumor.

g) Necrosis: Destrucción definida de áreas del

h) Quistes. Presencia de espacios quísticos en el componente estromal.

El seguimiento de los pacientes se realizó en policlínicos de cirugía y unidad de patología mamaria.

\section{RESULTADOS}

Las 39 pacientes del estudio fueron mujeres, con edad promedio 44.4 años con extremos de 20 y 66 años. Ninguna refirió antecedentes de carcinoma mamario. La detección del tumor fue en 37 $(94,8 \%)$ casos por autopalpación. En 25 (64,1\%) se localizó en mama derecha (16 en cuadrante superoexterno, 4 en cuadrante inferoexterno, 2 en región central, 2 en cuadrante superointerno y 1 comprometió toda la mama) y en 14 en mama izquierda ( 6 en cuadrante superoexterno, 2 en cuadrante inferoexterno, 3 en cuadrantes internos y 3 en región central).

Las características clínicas de los tumores filodes fueron:

A) Tamaño: Fluctuó entre 2 y $28 \mathrm{~cm}$, con un promedio de $8,3 \mathrm{~cm}$.

B) Consistencia: Dura en 29 pacientes, elástica 0 cauchosa en 6 , blanda en 2 y mixta en 1 .

C) Movilidad: Móvil en 35 pacientes, fija en 4 pacientes.

D) Dolor: Dolorosa en 6 pacientes e indolora en 33 pacientes. en 9 .

E) Superficie: Lisa en 30 pacientes e irregular

F) Crecimiento: Lento en 27 pacientes y rápido en 12.

Se efectuó mamografía a 27 casos observándose una imagen nodular redondeada en 20 , imagen lobulada en 2 y aumento localizado de la densidad en 5. En 13 se efectuó ecotomografía mamaria, 12 revelaron nódulos sólidos y la restante un nódulo mixto. En 20 se hizo citología por punción aspirativa que informó citología benigna en 8 , sospechosa de carcinoma en 4 , citología benigna compatible con tumor fibroepitelial en 5 y citología inflamatoria en 3.

Se planteó el diagnóstico clínico correcto de TF en $23(58.9 \%)$ pacientes, en 12 de fibroadenoma y en 4 de carcinoma.

Todas las enfermas fueron tratadas quirúrgica- mente, realizándose 29 mastectomías parciales, 7 mastectomías simples, 1 mastectomía simple extendida, 1 mastectomía de aseo y 1 mastectomía subcutánea. Los linfonodos axilares de la mastectomía simple extendida fueron histológicamente negativos.

Como procedimiento complementario a la cirugía inicial, en 4 casos en que se practicó mastectomía parcial, al conocerse el resultado de la histopatología, se realizó ampliación de márgenes ( 2 tumores malignos y 2 benignos). De las mastectomías simples se reconstruyó la mama en 4 mujeres, 3 con implantes mamarios y 1 con colgajo de músculo recto anterior.

Respecto a las características anatomopatológicas, de los 39 TF, 32 se catalogaron benignos, 3 borderline y 4 malignos. El tamaño promedio fue de $8,3 \mathrm{~cm}$ con rango de 2 a $28 \mathrm{~cm}$. En 31 casos los TF fueron bien circunscritos o lobulados y en 8 infiltrativos. Seis presentaron necrosis y hemorragia.

Los hallazgos microscópicos revelaron que todos los tumores presentaron características fibroepiteliales. En 4 el componente celular estromal presentó componente maligno, de tipo mesenquimatoso maligno. En 3 existió un grado de celularidad estromal densamente celular, intermedia en 13 y escasa en 23 casos. La actividad mitótica varió de menos de 1 a 5 mitosis por 10 campos de alto aumento, presentando un caso, 10 mitosis y otro 38 a 48. Se identificó sobrecrecimiento estromal en 11 casos, siendo en 4 de ellos un rasgo prominente. Se observó necrosis en 6 casos y quistes estromales en 8.

Dos de los 4 casos de TF malignos fueron sometidas a radioterapia complementaria y en otro se practicó metastasectomía pulmonar bilateral.

Las pacientes tienen seguimiento promedio de 70 meses, con extremos de 1 y 240 meses, existiendo 1 recidiva local. No se han presentado metástasis linfonodales axilares, tenemos un caso con metástasis pulmonares.

\section{DISCUSION}

Los TF de mama fueron descritos en 1938 por Johannes Müller denominándolos cistosarcoma filodes, por sus características microscópicas, siendo considerados tumores benignos hasta 1943 en que Cooper y Ackerman informaron sobre su potencial maligno ${ }^{5,7}$. Actualmente el término aceptado por la OMS es de tumor filodes ${ }^{10}$.

Corresponden a lesiones fibroepiteliales similares al fibroadenoma, del que se diferencian por su mayor celularidad y pleomorfismo del componente 
estromal. De acuerdo al aspecto morfológico de su componente mesenquimático se clasifican en benignos, potencialmente malignos (borderline) y malignos.

Nuestra serie está integrada exclusivamente por mujeres, no existiendo varones, los que son afectados en muy raras ocasiones, a veces asociados a ginecomastia ${ }^{1}$.

Se presentan generalmente entre los 35 y 55 años de edad $4,7,8$, lo cual concuerda con nuestra serie, donde el promedio de edad fue de 44 años.

La presentación clínica más frecuente fue una masa palpable, unilateral, detectada por autopalpación en el $94,8 \%$ de los casos, con predominio no significativo por la mama derecha. Las otras características destacadas fueron la consistencia dura o elástica, amplia movilidad, ausencia de compromiso de piel y de linfoadenopatías axilares.

El diagnóstico preoperatorio en los TF depende de la sospecha clínica, generalmente motivada por el tamaño del tumor y por su velocidad de crecimiento. En nuestra experiencia se planteó el diagnóstico basado en hechos clínicos en un 58,9\% de casos, destacando como principales diagnósticos diferenciales el de fibroadenoma en 12 casos y carcinoma en 4.

La evaluación de un tumor clínicamente sospechoso de TF usualmente contempla un estudio imagenológico. En nuestros casos la mamografía contribuyó al diagnóstico en 19 de 27 pacientes $(70,3 \%)$, porcentaje más alto de lo habitual ${ }^{10}$ destacando una imagen de gran tamaño, homogénea, de forma nodular, redondeada o lobulada con aumento de densidad, sin calcificaciones en su interior, lo cual es concordante con otras publicaciones ${ }^{2,5}$. Los hallazgos mamográficos pueden ser similares a los encontrados en fibroadenomas o carcinomas bien circunscritos. Al respecto, en las 8 pacientes restantes la mamografía orientó en 6 casos hacia fibroadenoma y en 2 a carcinoma mamario.

Las características de la ecotomografía mamaria, en las 13 pacientes en que el examen fue practicado, fueron concordantes con TF al evidenciar en 12 casos un nódulo sólido y en 1 nódulo con áreas quísticas, lo cual concuerda con la literatura revisada ${ }^{2,9,12}$.

La citología puede sugerir una lesión benigna en la mayor parte de los casos si se reúnen los elementos epiteliales y células bipolares, lo cual se apreció en la mayor parte de nuestros casos, sin embargo, este hallazgo no discrimina entre fibroadenoma y TF. El diagnóstico específico es planteable si se observan elementos estromales muy celulares además de grupos de células epiteliales con marcada hiperplasia. Esto se observó sólo en 5 de los casos que contaron con estudio citológico. Los 2 casos en que la citología sugirió carcinoma y resultaron lesiones benignas, se trataron de tumores con marcada celularidad estromal y componente epitelial hiperplásico con algunas atipias focales. Los problemas diagnósticos derivados del muestreo y de la escasa frecuencia de estos tumores dificultan la adquisición de destreza diagnóstica y hacen poco confiable la citología para casos específicos, lo que es avalado por la literatura ${ }^{13}$.

El diagnóstico definitivo de TF sólo se obtiene con el estudio histopatológico ${ }^{5,7}$. Según los parámetros estudiados se catalogaron como benignos 32 casos, borderline 3 y malignos 4 .

En todos los casos se realizó primariamente cirugía, considerada como la terapia idónea para los $\mathrm{TF}^{4,5,7,8,10,11}$. Previo a una operación es conveniente considerar que la mayoría de los TF son benignos, ya que no existen elementos clínicos que identifiquen los que resultarán ser malignos.

Cuando no existe sospecha clínica de un TF y se postula el diagnóstico de fibroadenoma, la cirugía habitual conlleva una resección del tumor por clivaje. Si la biopsia del espécimen revela un TF se recomienda reoperar para ampliar márgenes. En 4 pacientes en que ocurrió esta situación se amplió la cirugía y han evolucionado sin recidiva local. Si no se amplían los márgenes la recidiva aumenta 4 veces $^{2}$.

Si el diagnóstico es sospechado clínicamente, se recomienda realizar biopsia contemporánea. Si ésta corrobora dicho diagnóstico la extensión de la cirugía debe considerar la relación mama/ tumor, la cuantía de márgenes de tejido mamario sano para evitar recidivas y el aspecto cosmético resultante ${ }^{2}$.

Es posible practicar una mastectomía parcial con margen de tejido sano de $1-2 \mathrm{~cm}$ tanto en tumores pequeños ${ }^{8}$ como de mayor tamaño si es dable conservar la mama estéticamente aceptable. Cuando esto no es factible se opta por la mastectomía simple ${ }^{5}$ idealmente seguida de reconstrucción mamaria. Las 6 pacientes en que realizamos mastectomía simple la indicación fue sustentada por el gran tamaño tumoral.

La linfadenectomía axilar en los TF malignos es controversial puesto que las metástasis habitualmente se producen por vía hematógena. En casos muy seleccionados, en que clínicamente se evidencia un cáncer mamario, apoyado por citología y biopsia, es planteable realizar una mastectomía simple extendida o radical modificada ${ }^{9}$.

La cirugía conservadora es la más corriente en los TF, lo que motiva un reducido número de com- 
plicaciones post operatorias, que usualmente son menores y de fácil manejo.

Los 2 casos de nuestra serie con TF maligno recibieron radioterapia en el lecho mamario, conducta que es sugerida por algunos autores. Las pacientes han evolucionado sin recidiva local.

Actualmente tenemos un caso de metástasis pulmonares bilaterales que han sido sometidas a metastasectomía y está planificado realizar quimioterapia adyuvante.

Nuestras pacientes tienen un seguimiento promedio de 70 meses, con un rango de 1 a 140 meses. Existe 1 caso de recidiva local la que es descrita hasta en un 20 a $30 \%$ de los $\operatorname{TF}^{1,14}$ y otro de metástasis sistémica. En 19 pacientes el seguimiento es mayor de 3 años.

Los factores histológicos clásicamente asociados a recurrencia tumoral y a metástasis sistémicas son el margen tumoral, el sobrecrecimiento estromal, el número de mitosis y la presencia de atipias celulares ${ }^{4}$, siendo el principal predictor el sobrecrecimiento estromal. De los 4 casos en que se identificó sobrecrecimiento estromal ${ }^{10}$ uno de ellos presentó metástasis a distancia a los 6 meses de seguimiento. Del grupo de pacientes en que se identificó más de una mitosis por HPF uno de ellos presentó metástasis a los 6 meses de seguimiento. De las 6 pacientes en que se identificó margen infiltrativo una de ellas presentó recidiva local a los 23 meses y la otra evolucionó con metástasis pulmonares a los 6 meses.

La citometría de flujo en el análisis de ploidía y fase $\mathrm{S}$ como predictor de recurrencia tiene un escaso rendimiento ${ }^{1}$. Algunos autores reportan aneuploidía en $75 \%$ de los casos benignos y $50 \%$ de los malignos ${ }^{1}$. Una fase S lenta fue encontrada en $70 \%$ de los tumores que no recurrieron y en $80 \%$ de los que recurrieron ${ }^{1}$. Otros autores señalan que una asociación entre aneuploidía y alto índice de proliferación celular, predispone a una evolución clínica adversa $^{15}$. En nuestro hospital no se realiza este tipo de detección.

La caracterización de los pacientes de nuestra serie revela que los TF son poco frecuentes, cuyo diagnóstico se postula ante un tumor voluminoso, móvil, consistencia dura o elástica sin compromiso de linfoganglios regionales. El estudio imagenológico es útil para respaldar la sospecha clínica o sugerir un diagnóstico distinto. La citología no fue de gran utilidad siendo el estudio histopatológico el que proporcionó el diagnóstico específico y el genio biológico del tumor. Este estudio histopatológico precisó el grado de sobrecrecimiento estromal, número exacto de mitosis y el margen tumoral en la pieza operatoria, factores relacionados con la pro- babilidad de metástasis y con el pronóstico de la paciente.

El tratamiento de los TF de nuestra serie fue quirúrgico, resecando el tumor con un margen de tejido sano. En la mayoría se realizó cirugía conservadora puesto que existía una relación mama/tumor favorable. Como a otros autores ${ }^{16,17}$ resultó manifiesto que los márgenes quirúrgicos libres aparecen como el factor primordial para evitar recidiva local.

En los TF de alto grado el uso de radioterapia complementaria disminuiría la recidiva local ${ }^{17}$ y es incierto el rol de la quimioterapia por lo cual es indispensable conocer el resultado de mas estudios para obtener conclusiones definitivas respecto a estas medidas terapéuticas.

\section{REFERENCIAS}

1. Rose'n PP: Fibroepitelial neoplasm. In: Rosen's Breast Pathology. Philadelphia, Lippincott-Raven Publishers 1997; 143-75.

2. Martínez V, Torres S, Díaz P: Tumor Phylloides. En: Pabst $Y(e d)$, Patología mamaria benigna. Santiago, Fundación de Investigación y Perfeccionamiento Médico 1994; 97-103.

3. Bernstein L, Deapen D, Ross R. The descriptive epidemiology of malignant cystosarcoma phyllodes tumors of the breast. Cancer 1993; 71: 3020-3024.

4. Reinfuss M, Mitos J, Duda K, Stelmach A, Rys J, Smolak K. The treatment and prognosis of patients with phyllodes tumor of the breast. Analysis of 170 cases. Cancer 1996; 77: 910-916.

5. Pierat J, Burmeister R, Steinberg J, Schalper J. Tumor filodes de la mama: Diagnóstico y tratamiento en 26 casos. Rev Chil Cir 1992; 44: 52-56.

6. Hawkins R, Schofield J, Fisher C, Wiltshaw E, McKinna A. The clinical and histologic criteria that predict metastases from cystosarcoma phyllodes. Cancer 1992; 69: 141-147.

7. Uribe A, Bravo G, Uribe A, Viada R, Capetillo M, Villarroel T. Tumor filodes: diagnóstico y tratamiento. Rev Chil Obstet Ginecol 1995; 60: 17-22.

8. Chaney A, Pollack A, Mcneese M, Zagars G, Pisters $P$. Primary treatment of cystosarcoma phyllodes of the breast. Cancer 2000; 89: 1502-1511.

9. Pérez JA., Poblete MT, Santolaya R. Tumor filodes mamario. Una revisión clinicopatológica de 14 casos. Rev Méd Chile 1995; 123: 1379-1386.

10. Salvadori B, Cusumano F, Del Bo R, Delledonne V, Gras M. Surgical treatment of phyllodes tumor of the breast. Cancer 1989; 63: 2532-2536.

11. Cohn-Cedemark G, Rutqvist L, Rosendahl I, Silfverswärd C: Prognostic factors in cystosarcoma pyllodes. A clinicopathology study of 77 patients. Cancer 1991; 68: 2017-2022.

12. Stelling $C$, Powel D: Masas mamarias circunscritas. En: Powel D, Stelling C (eds), Enfermedades de la 
mama. Diagnóstico y detección. Madrid, MosbyDoyma, 1995; 159-191.

13. Shimizu K, Masawa N, Yamada T, Okamoto K, Kanda K. Cytologic evaluation of phyllodes tumors as compared to fibroadenomas of the breast. Acta Cytol 1994; 38: 891-897.

14. De Roos W, Kaye P, Dent D. Factors leading to local recurrence or death after surgical resection of phyllodes tumours of the breast. Br J Surg 1999; 86: 396-399.

15. El Nagara AK, Ro JY, Mclemore D. DNA content and proliferative activity of cystosarcoma phyllodes of the breast. Am J Clin Pathol 1990; 93: 480-485.

16. Pandey M, Mathew A, Kattoor J, Abraham E, Mathew B. Malignant phyllodes tumor. Breast J 2001; 7: 411416. 